\title{
Towards Acoustic Radiation Free Lamb Wave Resonators for High-resolution Gravimetric Biosensing
}

\author{
Feng Gao, Member, IEEE, Aisha M. Al-Qahtani, Abdelkrim Khelif, Farid Boussaid, Sarah Benchabane,Yu \\ Cheng, Omar El Agnaf and Amine Bermak Fellow, IEEE
}

\begin{abstract}
A novel Lamb wave resonator with fully suppressed acoustic radiation in water is proposed for high-resolution masssensitive detection of biomolecules. The elimination of acoustic radiation is achieved by slowing down the Lamb wave to a velocity lower than the sound speed in water. This enables high-qualityfactor resonance in water and reduces the sensing frequency noise. High aspect ratio electrodes (HAREs) are used to slow down the Lamb wave. The elastic resonance and large surface area of the HAREs can also enhance the mass sensitivity of the device. The improved mass sensitivity together with the low frequency noise substantially improves the overall sensing resolution. Although reducing the plate thickness can also slow down the Lamb wave, it makes the device very fragile and not practical to use. In contrast, slowing down the Lamb wave by increasing electrode height allows the use of thick plates which is robust. In this paper, the behavior and performance of the proposed high aspect ratio electrode Lamb wave resonator (HARE-LWR) are theoretically analyzed using finite element method simulations. Optimum design parameters were found through the simulations. Reported results show that a significant figure of merit improvement was achieved by the proposed HARE-LWR design.
\end{abstract}

Index Terms-Acoustic resonators, Biosensors, Lamb wave, Ultrasonic transducers

\section{INTRODUCTION}

$\mathbf{T}$ HE in-situ detection of biomolecules is important in applications such as disease diagnosis and food security analysis [1]-[3]. A key requirement in achieving insitu biosensing is to miniaturize the sensor while keeping a good sensitivity. Among various transduction techniques, mass-sensitive acoustic biosensors are popular because of their small size, fast response and high sensitivity [4], [5]. Quartz crystal microbalance (QCM) [6]-[10], film bulk acoustic wave resonators (FBAR) [11]-[13] and surface acoustic wave (SAW) [14]-[16] sensors are the three main types of acoustic biosensors that have been extensively studied.

F. Gao, A. M. Al-Qahtani and A. Bermak are with the College of Science and Engineering, Hamad Bin Khalifa University, Doha, Qatar. (e-mail: fgao@hbku.edu.qa; aialqahtani@hbku.edu.qa; abermak@hbku.edu.qa).

A. Khelif and S. Benchabane are with Institut FEMTO-ST, CNRS, Université de Bourgogne Franche-Comté, 25000 Besançon, France. (email: abdelkrim.khelif@femto-st.fr; sarah.benchabane@femto-st.fr).

F. Boussaid and also F. Gao are with the school of Department of Electrical, Electronic and Computer Engineering, the University of Western Australia, Perth, Australia. (e-mail: farid.boussaid@uwa.edu.au).

Y. Cheng is with the Department of Civil and Environmental Engineering, the Hong Kong University of Science and Technology, Hong Kong. (e-mail: ychengad@connect.ust.hk).

O. E. Agnaf is with the Qatar Biomedical Research Institute, Hamad Bin Khalifa University, Doha, Qatar. (e-mail: oelagnaf@hbku.edu.qa).
Despite its relatively low sensitivity, QCM is the most popular mass-sensitive biosensor due to its simplicity and low-cost. It has become a reference tool for label-free detection of biomolecules [17]. Shear mode FBAR biosensor shares the same principle as QCM but works at a much higher frequency due to its small film thickness. The high operating frequency significantly improves its mass sensitivity. However, it is more costly than QCM due to its more complex fabrication process. SAW is reported to be the most sensitive acoustic sensor in gas sensing [18], [19]. This is because SAW is very sensitive to the surface changes as the energy is mostly confined around the surface. Nevertheless, applying SAW in biosensing is difficult due to the strong acoustic radiation in water. Shear horizontal SAW (SH-SAW) and love SAW sensors were developed to reduce the acoustic radiation and thus enable operation in water [20]. Both sensors rely on the principle that particle movement of shear-waves are parallel to the water interface and thus less energy is transferred to water compared to longitudinal waves. In fact, QCM and FBAR also suffer from acoustic radiation in water. They mitigate the problem also by making the device work in shear wave mode. Although the shear wave can reduce acoustic radiation, the corresponding resonance quality factors are still significantly lower in water compared to the ones in air.

One potential way to fully suppress the acoustic radiation in 
water is to reduce the acoustic wave velocity to a value below the radiation limit, which is the sound speed of water. This idea was exploited in Lamb wave biosensors with very thin plates [21]-[24]. It was found that reducing the plate thickness can reduce the velocity of antisymmetric Lamb wave and thus suppress the acoustic radiation. However, to fully suppress the radiation, the plate thickness needs to be a few percents of the wavelength, which makes the device very fragile especially when working in water. Due to this reason, the thin plate Lamb wave biosensor has rarely been further studied. Besides the plate thickness reduction method in Lamb wave based sensors, increasing the electrode height was also reported to be effective in reducing the velocity of Rayleigh SAW in air [25]. By applying the latter method to Lamb wave, we found that Lamb wave can also be slowed down by increasing the electrode height in both air and liquid environment. If the electrode is thick enough, the velocity of the Lamb wave can be reduced to below the sound velocity of water for which the acoustic radiation is fully suppressed. Because this method can be implemented with thick plates, it makes the device much more robust than the thin plate Lamb wave resonator, enabling broad practical applications of the device. In this paper, we analyzed the behavior and performance of the proposed biosensor based on high aspect ratio electrode Lamb wave resonator (HARE-LWR) through finite element method (FEM) simulations. The results aim to serve as a fundamental design reference for future experimental implementations.

The rest part of this paper is organised as follow. The conventional approach for slowing down Lamb wave through reducing the plate thickness is firstly discussed in Section II. The design of the HARE-LWR is then introduced to overcome the reliability problem of the plate thickness reduction method. In section III, the performance of the HARE-LWRs under different plate thickness, electrode height and electrode material configurations is analysed and compared by their mass sensitivity, resonance quality factor, ectromechanical coupling factor and figure of merit.

\section{Device Design and Analysis for Slow Lamb WAVE}

HARE-LWR achieves the reduced phase velocity by increasing the electrode height. In comparison, the phase velocity of an conventional antisymmetric mode $\left(A_{0}\right)$ Lamb wave resonator is reduced by decreasing its plate thickness [21]. These two devices can both achieve in-liquid operation once their phase velocity is lower than the sound velocity of water. In this section, the difference between the slowing down effects of the HARE-LWR and the conventional lamb wave resonator are studied by FEM simulation.

\section{A. FEM modeling parameters}

Fig. 1 shows the FEM simulation unit cells of the conventional lamb wave resonator and the HARE-LWR. The widths of both unit cells are set to one wavelength $(\lambda)$ with periodic conditions applied to the two sides of the unit cells. The periodic conditions are equivalently repeating the unit cells in the propagating direction, making the number of interdigitated

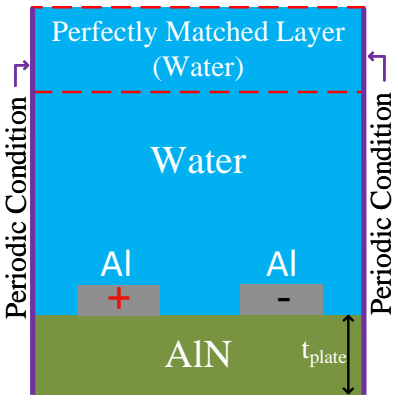

(a)

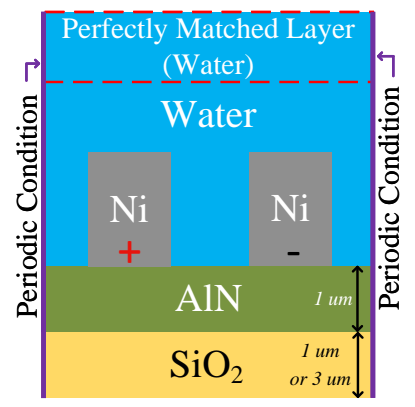

(b)
Fig. 1. FEM simulation unit cell (cross-section) of (a) the conventional Lamb wave resonator and (b) the HARE-LWR.

transducer (IDT) pairs infinite. The actual FEM simulation is performed with 3D models. The depths of the 3D models are set to $0.2 \lambda$ with periodic boundary conditions applied to the front and back sides, which equivalently extends the model depths (acoustic aperture) to infinity. The wavelengths of both resonators are set to $10 \mu \mathrm{m}$. This value is chosen because it is a frequently used dimension for microfabrication with the classic photolithography method. In fact, the selection of simulation dimension does not affect the final conclusion as all the numerical calculations are scalable. On top of the devices operation environment, which is water or air. The depth of the environment is set to $4 \lambda$ for both devices with the top $1 \lambda$ set to perfectly matched layer (PML). The PML adsorbs all the acoustic wave propagates into it, which is equivalent to extend the depth of the environment to infinity.

The conventional lamb wave resonator (Fig. 1a) is made of an aluminum nitride (AIN) plate with 200-nm-thick aluminum IDTs on top. One of the IDT finger is set to 1 volt amplitude AC electrical potential while the other is set to ground to form the electrical stimulation. The width and separation of the IDT electrodes are both half wavelength. The thickness of the AlN plate $\left(t_{\text {plate }}\right)$ is set as the sweeping parameter in the simulation to study the slowing down effect. The HARE-LWR (Fig. 1b) is made of 1- $\mu m$ AlN nitride film with $1-\mu m$ or 3- $\mu m$ silicon dioxide $\left(\mathrm{SiO}_{2}\right)$ supporting layer underneath it. High aspect ratio IDTs were placed on top of the AIN for generating the hybridized slow wave. The electrical stimulation, IDT width and separation are set the same as the conventional lamb wave resonator in Fig. 1a. The height of the high aspect ratio electrode (HARE) is set as sweeping parameter to study the slowing down effect. The material of the metal electrodes is changed among nickel $(\mathrm{Ni})$, gold $(\mathrm{Au})$, copper $(\mathrm{Cu})$ and platinum $(\mathrm{Pt})$ to study the relevant impact on the device performance.

Multiple physics are involved in the simulation for the two devices. Pressure acoustics are solved in the water/air environment while solid mechanics are solved in the substrate plate and metal electrodes. Electrostatic study is applied to the whole device except the metal electrodes part. Piezoelectric effect is considered in the AlN part. Acoustic-structural boundary is applied to the interface between the device and the water/air environment to couple the pressure acoustic and solid mechanics study. 


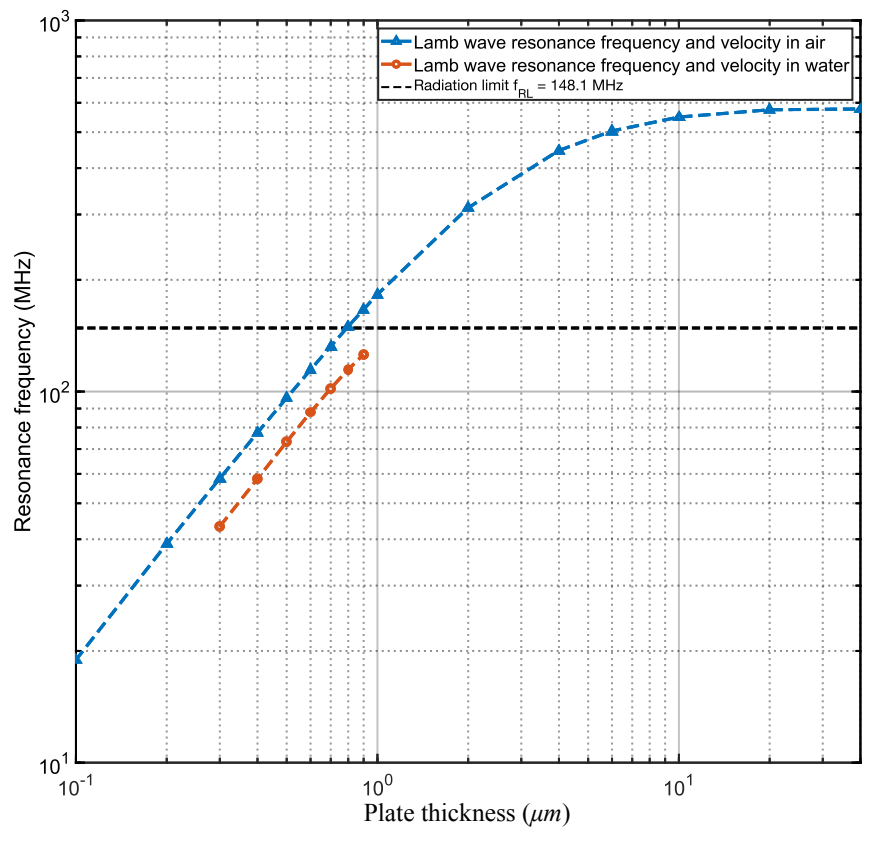

Fig. 2. The resonance frequency of the $\boldsymbol{A}_{\mathbf{0}}$ mode lamb wave resonator changing with plate thickness in air and in water. The black line corresponds to the frequency of the radiation limit in water.

\section{B. Slowing down lamb wave by reducing plate thickness}

Fig. 2 shows the simulated resonance frequency of the $A_{0}$ mode Lamb wave changing with the plate thickness in air and in water, respectively. The blue line in Fig. 2 shows that the Lamb wave velocity reduces with the decrease of the plate thickness $\left(t_{\text {plate }}\right)$ in air. At large plate thicknesses, the wave transforms into a Rayleigh wave and propagates at the velocity of Rayleigh wave, which is about $5760 \mathrm{~m} / \mathrm{s}$. The resonator remains operational in water only when the Lamb wave velocity is slower than the sound velocity in water (radiation limit), as depicted by the orange line in Fig. 2. This happens when the plate thickness is smaller than $0.09 \lambda$. The black line marks the frequency of the radiation limit $\left(f_{R L}=148.1 \mathrm{MHz}\right)$, which corresponds to a wave velocity equals to the sound speed of water. The resonance frequencies of the resonators are lower in water than in air because of the mass loading of water. At plate thicknesses smaller than $0.02 \lambda$, the resonance also disappears in water, possibly due to the insufficient piezoelectric conversion at very small plate thicknesses [26]. The acoustic pressure distribution of the $0.05 \lambda$-plate resonator at its resonance frequency $(73.3 \mathrm{MHz})$ in water confirms the suppression of acoustic radiation (Fig. 3a). It can be seen that the positive (red part) and negative (blue part) acoustic pressures only exist in a small region above the resonator, which means the acoustic energy is confined in this small region. For comparison, the acoustic pressure distribution of the Lamb wave resonator with $0.2 \lambda$ thickness is shown in Fig. $3 b$. The alternating positive and negative acoustic pressures extend all the way up till they get absorbed by the PML. This means that the acoustic energy is strongly radiative. It should be noted that a pseudo-resonance frequency $(280 \mathrm{MHz})$ was selected to show the radiation of the $0.2 \lambda$ -

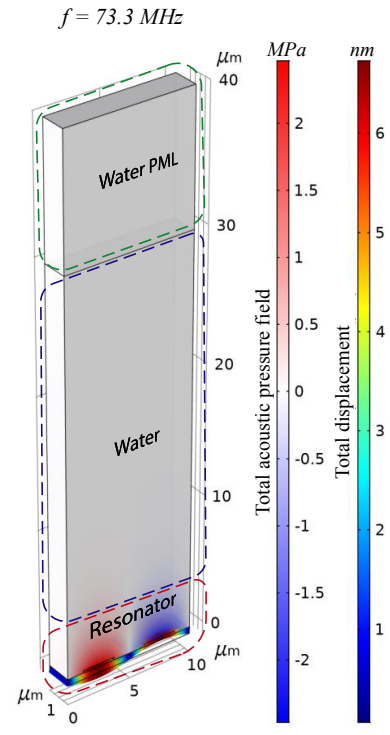

(a)

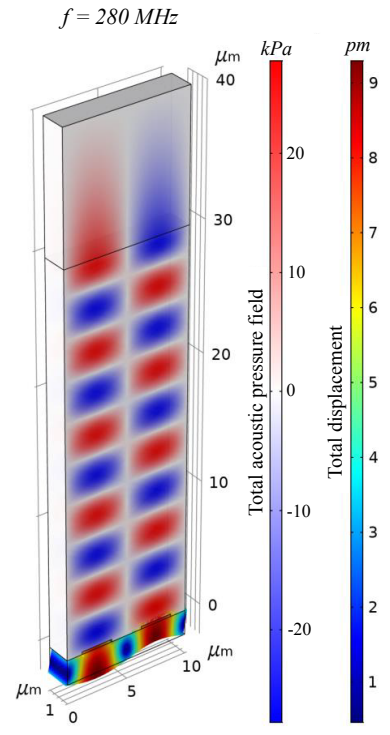

(b)
Fig. 3. (a) Lamb wave resonator with $0.05 \lambda$ plate thickness showing resonance with fully suppressed acoustic radiation in water. (b) Lamb wave resonator with $0.2 \lambda$ plate thickness showing resonance with strong acoustic radiation in water.

plate device as it cannot resonate in water. This frequency is calculated by subtracting the estimated frequency shift caused by water loading from the resonance frequency of the device in air.

Although the Lamb wave resonator can work in water when the plate is sufficiently thin, it is very fragile and difficult to fabricate. As shown in Fig. 2, to have resonance in water, the plate thickness of the Lamb resonator needs to be smaller than $0.09 \lambda$. To have a good quality factor, the acoustic aperture and number of IDT pairs should be at least $20 \lambda$ and 20 pairs, respectively [27]. The surface area of the film is thus at least $20 \lambda \times 20 \lambda$. Considering the internal stress of the film and other imperfections during fabrication, the yield of this large-area thin device will be very low. Although higher plate thickness can be used when the wavelength is large [21], the aspect ratio (the plate thickness relative to the wavelength and film area) remains the same and thus the structural strength remains the same. Due to their fragility, these ultra thin plate Lamb wave resonators are rarely reported in biosensing applications.

\section{Slowing down lamb wave by HARES}

To overcome the limitations of the plate thickness reduction technique, we propose the HARE-LWR that uses HAREs for slowing down the lamb wave without sacrificing the structural strength of the device. The mass loading caused by the HAREs together with the hybridization between the elastic resonance of the HAREs and the lamb wave resonance can significantly reduce the phase velocity of the HARE-LWR.

Fig. 4 shows the FEM simulated electrode-height-dependent resonance frequency (slowing down curve) of two Ni-electrode Lamb wave resonators with $2-\mu m$ and $4-\mu m$ plate thicknesses. The use of two plate thicknesses is to investigate the impact 


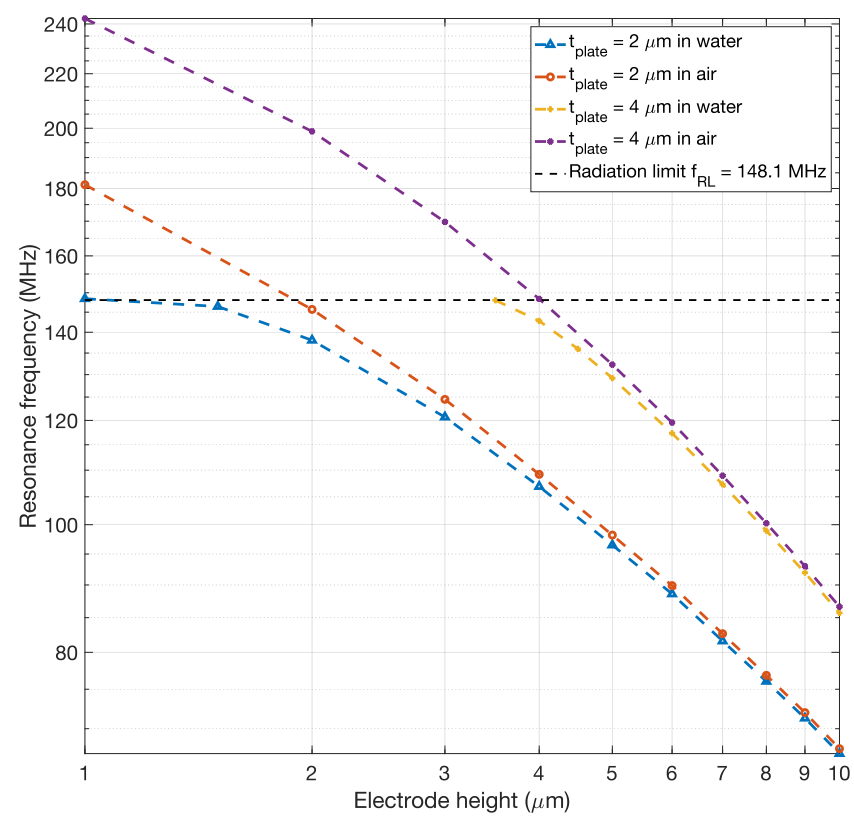

Fig. 4. The slow-down curves of the Ni-electrode HARE-LWR with 2 and $4 \mu \mathrm{m}$ plate thickness. The black line corresponds to the frequency of the radiation limit in water.

of plate thickness on the slowing down effect. The two values are chosen based on the typically available $\mathrm{SiO}_{2}$ thicknesses of an oxidized silicon wafer, which are 1 and $3 \mu \mathrm{m}$. Thicker $\mathrm{SiO}_{2}$ is also possible but will take a lot longer to grow on the silicon wafer, so they are not considered. The slowing down curve of the HARE-LWR with gold, copper and platinum electrodes are similar to that of nickel and are shown in Fig. S1-S3 of Supporting Information. It can be seen that the 2$\mu m$-plate resonator remains operational in water even when its electrode height is only $1 \mu \mathrm{m}$. However, the electrode height needs to increase to more than $2 \mu \mathrm{m}$ for the acoustic radiation to be sufficiently suppressed. This is revealed by the acoustic pressure distribution of the resonators when their electrode height increases. In Fig. 5a, the positive acoustic pressure (red part) and negative acoustic pressure (blue part) of the $1-\mu m$-electrode resonator at its resonance frequency (148.5 MHz) extend up to the PML boundary with gradually decreasing intensities. Although the amount of acoustic energy reaching the PML boundary is much smaller than that of the conventional Lamb wave resonator shown in Fig. 3b, the acoustic energy confinement is still not very effective. In contrast, the acoustic energy of the $2-\mu m$-electrode resonator at its resonance frequency $(138.1 \mathrm{MHz})$ is limited in the region about $2 \lambda$ high above the electrodes (Fig. 5b). The $2 \lambda$ confinement length is an estimation of the region where the acoustic pressure fields marked by red and blue are clearly visible. The resonators with $4-\mu m$ plate thickness show similar behavior. The radiation is seen to be strong at its resonance frequency $(148.1 \mathrm{MHz})$ when its electrode height is $3.5 \mu \mathrm{m}$ (Fig. 5c). In contrast, when its electrode height increases to 5 $\mu m$ (Fig. 5d), the radiation at its resonance frequency (129.2 $\mathrm{MHz}$ ) is suppressed and the energy gets confined in the region about $1.5 \lambda$ from the electrodes. The relatively strong radiation of the resonators in Fig. 5a and $5 \mathrm{c}$ is due to the fact that their wave velocity is close to the sound speed of water. It is thus necessary to further increase the electrode height to leave a margin of about $100-200 \mathrm{~m} / \mathrm{s}$ between the wave velocity of the resonator and the sound speed in water so that the acoustic radiation can be fully suppressed. In Fig. 4, the resonance frequencies of the two resonators in air are also shown for comparison. Comparing the results of Fig. 4 and Fig. 2, it can be found that the frequency shifts due to the mass loading of water in HARE-LWRs are much smaller than those in the conventional Lamb wave resonators. This smaller mass loading effect of water can potentially make the HARELWRs more resistant to environmental disturbances in water.

\section{Performance of the hare-LWR for BIOMOLECULE DETECTION}

The mass sensing resolution $\left(R_{m}\right)$ or the detection limit (LOD) of the frequency based biosensors are determined by the following equation:

$$
R_{m}=\frac{S_{m}}{n_{f}}
$$

where $S_{m}$ and $n_{f}$ are the mass sensitivity and the frequency noise (jitter) of the sensor, respectively. To maximize the sensing resolution, the sensor should have both high mass sensitivity and low frequency noise.

\section{A. Mass sensitivity of the HARE-LWR}

The mass sensitivity of the HARE-LWR was analyzed by FEM simulation. A 200-nm polymer layer (Polymethyl methacrylate, PMMA) was used as the mass sensing layer. It uniformly covers the surface of the HARE-LWR as shown in the insert of Fig. 6a. To evaluate the mass sensitivity of the device, we changed the density of the sensing layer and recorded the corresponding frequency shift. The variation of the density is an emulation of the mass loading effect when the sensor absorbs biomolecules. The mass sensitivity is calculated by the following equation:

$$
S_{m}=\left|\frac{\Delta f}{t \Delta \rho}\right|
$$

where $\Delta f$ is the frequency shift before and after mass loading, $t$ is the thickness of the sensing layer, $\Delta \rho$ is the density variation of the sensing layer. Fig. 6a shows the simulated electrode-height-dependent mass sensitivities of the HARELWRs with different electrode materials and plate thicknesses. The mass sensitivity of all the devices exhibit similar behavior. After the electrode height exceed the limit which the devices can operate in water, the mass sensitivities of all the devices increase rapidly with the increasing of the electrode height and reach maximum points. A gradual decrease is then observed with the further increase of the electrode height. Table I summarises the maximum mass sensitivities $S_{m-\max }$ of all HARE-LWR configurations and their corresponding electrode height $\left(h_{\text {ele-max }}\right)$ and operating frequency $\left(f_{\max }\right)$.

Among the four analysed electrode material, $\mathrm{Cu}$-electrode HARE-LWR has the best mass sensitivity which is closely 


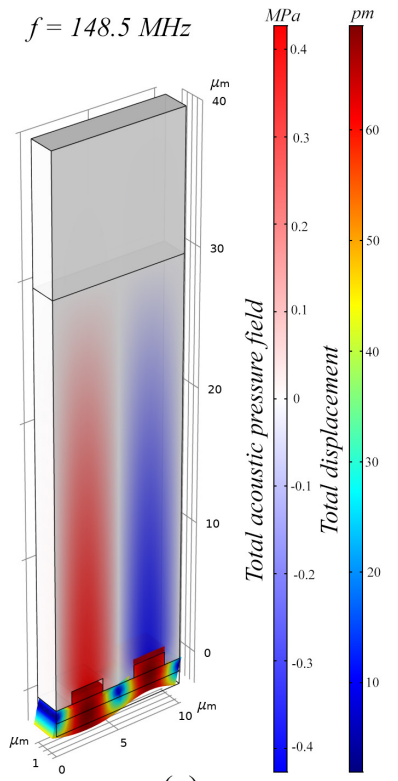

(a)

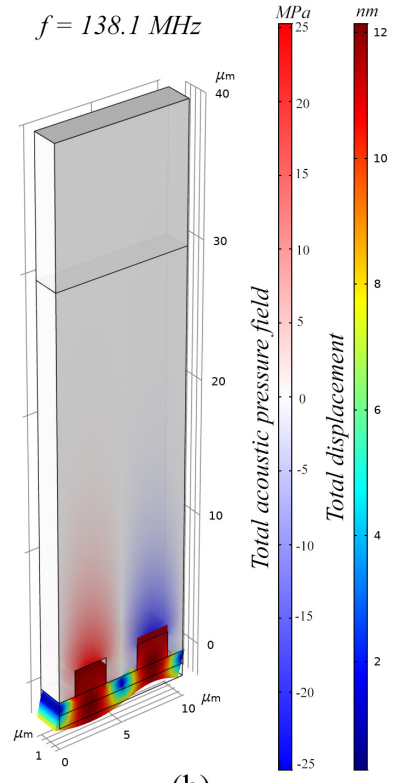

(b)

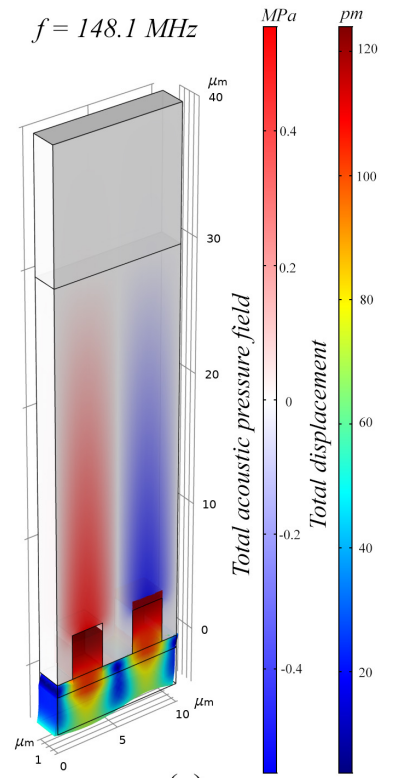

(c)

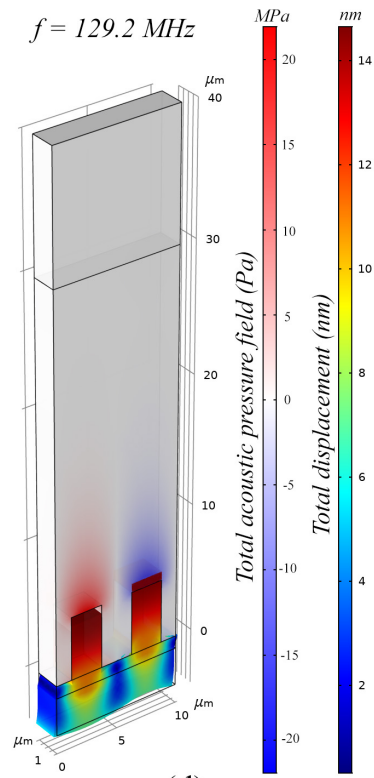

(d)

Fig. 5. (a-b). Displacement (enlarged) and acoustic pressure distribution of the 2- $\boldsymbol{\mu} \boldsymbol{m}$-plate Ni-electrode HARE-LWR at 1 and $2 \boldsymbol{\mu m}$ electrode height. (c-d). Displacement (enlarged) and acoustic pressure distribution of the $4-\mu m$-plate Ni-electrode HARE-LWR resonator at 3.5 and $5 \mu m$ electrode height.

TABLE I

MAXIMUM MASS SENSITIVITY OF THE HARE-LWRS

\begin{tabular}{|c|c|c|c|c|}
\hline $\begin{array}{c}\text { Electrode } \\
\text { Material }\end{array}$ & $\begin{array}{c}t_{\text {plate }} \\
(\mu \mathrm{m})\end{array}$ & $\begin{array}{c}S_{m-\max } \\
\left(\mathrm{Hz} /\left(\mathrm{ng} \cdot \mathrm{cm}^{2}\right)\right)\end{array}$ & $\begin{array}{c}h_{\text {ele-max }} \\
(\mu \mathrm{m})\end{array}$ & $\begin{array}{c}f_{\max } \\
(\mathrm{MHz})\end{array}$ \\
\hline $\mathrm{Ni}$ & 2 & 62.2 & 2.2 & 129.96 \\
$\mathrm{Ni}$ & 4 & 62.4 & 4.6 & 132.32 \\
$\mathrm{Au}$ & 4 & 39.1 & 2 & 132.20 \\
$\mathrm{Cu}$ & 4 & 63.9 & 4 & 130.09 \\
$\mathrm{Pt}$ & 4 & 34.5 & 2 & 131.79 \\
\hline
\end{tabular}

followed by the Ni-electrode HARE-LWR. In comparison, HARE-LWRs based on gold and platinum are less sensitive. For the nickel electrodes, the mass sensitivities of two HARELWRs with different plate thicknesses were simulated. They showed almost the same maximum mass sensitivity, but the 4$\mu m$-plate HARE-LWR reaches the maximum sensitivity at a larger electrode height of $4.6 \mu \mathrm{m}$ compared to the $2.2 \mu \mathrm{m}$ electrode of the 4- $\mu m$-plate HARE-LWR. This is because the original phase velocity of the 4- $\mu m$-plate HARE-LWR is faster than that of the 2- $\mu m$-plate HARE-LWR, it thus requires higher electrodes to sufficiently slow down its phase velocity. Despite the electrode material and height differences of the HARE-LWRs, it is interesting to find that all the devices reaches the maximum mass sensitivity at similar operating frequencies around $130 \mathrm{MHz}$. This phenomenon suggest that we can change the electrode configuration to improve the device reliability or adapt it to sensing applications that have requirement on electrode material while keeping the same sensitivity at the same operating frequency.

To investigate the origin of the high mass sensitivity of the HARE-LWR, we simulated the mass sensitivity of the $\mathrm{Cu}$ electrode HARE-LWR when the sensing film coverage on the device surfaces changes. The plate thickness and electrode height of the HARE-LWR are both $4 \mu \mathrm{m}$. The frequency shifts of the impedance curves together with the sensing layer coverage diagram are shown in Fig. S4 of supporting information. The mass sensitivities of the device with sensing film covering all the surface, the electrode surface and the bottom surface are 63.87, 62.61 and $2.78\left(\mathrm{~Hz} /\left(\mathrm{ng} \cdot \mathrm{cm}^{2}\right)\right)$, respectively. This means the high mass sensitivity of the HARE-LWR is mainly contributed by the elastic resonance of the HAREs. The contribution from the mass loading of the bottom surface is very small.

\section{B. Quality factor and FOM of the HARE-LWR}

To determine the resolution, we also need to evaluate the frequency noise of the sensor. However, the frequency noise is a parameter associated to the complete sensing system. It is affected by the noise from the detection circuits. We thus use the quality factor of the resonator to evaluate the frequency noise caused by the sensor part. Resonators with higher quality factors will exhibit a lower frequency noise of the final sensing system. The quality factor calculated from the simulated electrical impedance is given by the following equation:

$$
Q=\frac{f}{2} \frac{d \angle Z}{d f}
$$

where $f$ and $Z$ are the working frequency and electrical impedance of the device respectively. Fig. $6 \mathrm{~b}$ shows the calculated quality factor of the HARE-LWRs at different electrode heights. For all the HARE-LWRs, the quality factor is relatively low at small electrode heights due to the incomplete suppression of the acoustic radiation. When the electrode height further increases, the radiation is fully eliminated and thus the quality factor improves dramatically. After the rapid quality factor improvement, the quality factors follow different trends depending on the plate thickness and electrode material. For 


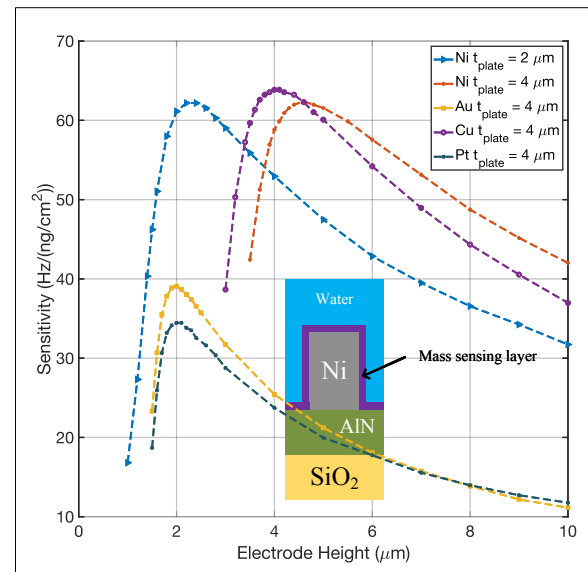

(a)

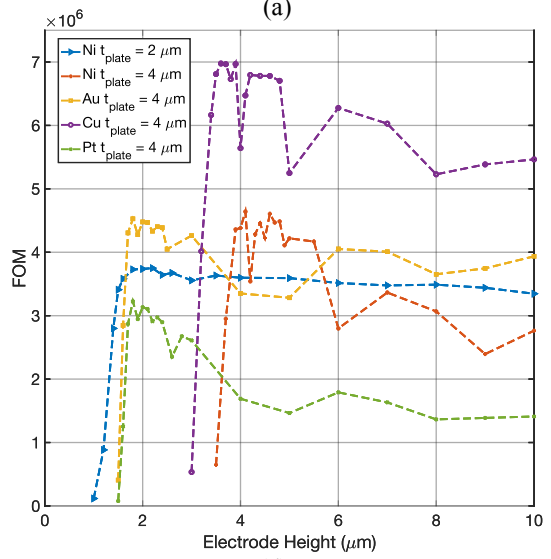

(d)

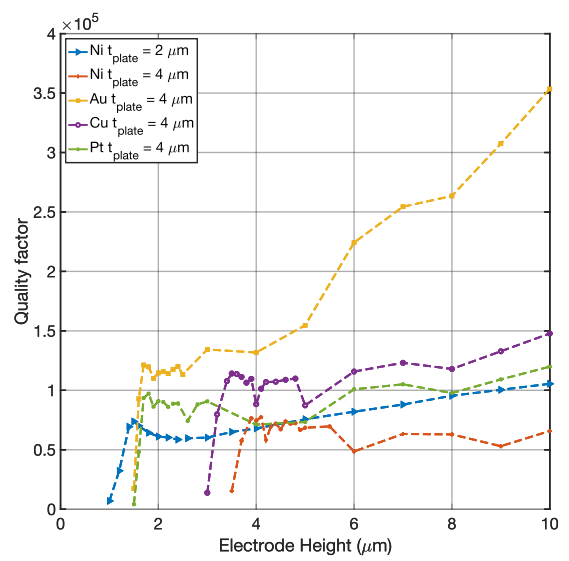

(b)

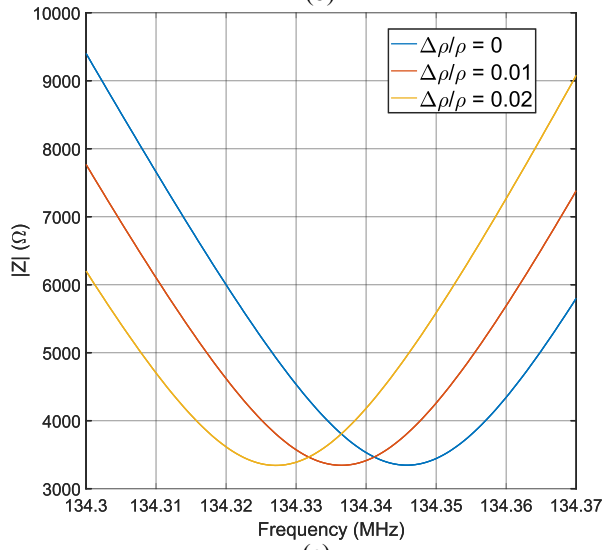

(e)

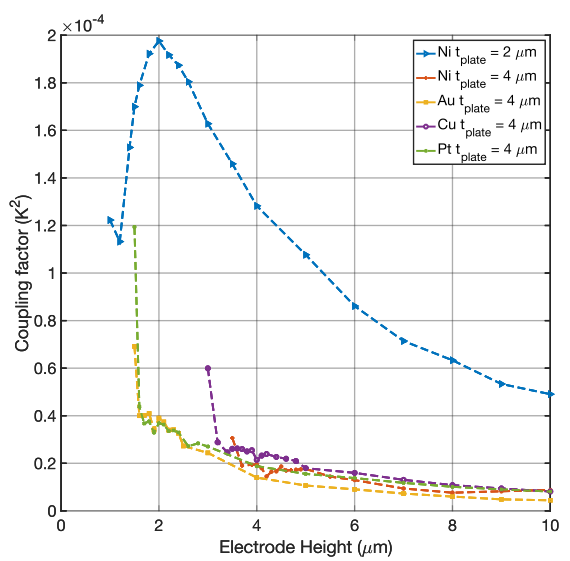

(c)

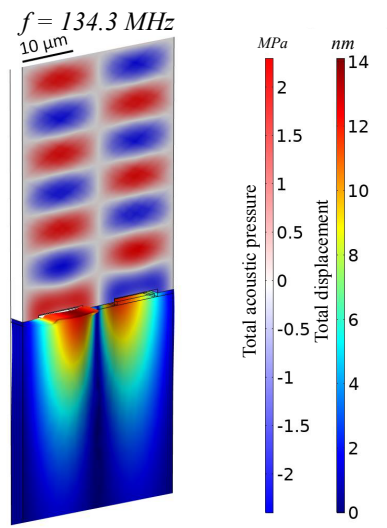

(f)

Fig. 6. (a) Mass sensitivity $\left(\boldsymbol{S}_{\boldsymbol{m}}\right)$, (b) Quality factor $(\boldsymbol{Q})$, (c) Electromechanical coupling factor and (d) Figure of merit $\left(\boldsymbol{F} \boldsymbol{O} \boldsymbol{M}=\boldsymbol{S}_{\boldsymbol{m}} \times \boldsymbol{Q}\right)$ of the HARE-LWR biosensors. The insert in 5 a shows how the mass sensing layer covers the surface of the HARE-LWR biosensor. (e). Resonance frequency shift of the SH-SAW biosensor based on $36^{\circ} \mathrm{Y} \mathrm{LiTaO}_{3}$. (f) Acoustic radiation of the SH-SAW biosensor at resonance frequency.

the 2- $\mu m$-plate Ni-electrode HARE-LWR, the quality factor decreases slightly and then increases again with the increasing of the electrode height. For the 4- $\mu m$-plate HARE-LWR based on nickel, copper and platinum, the quality factor saturates and fluctuates with the increasing of electrode heights. The quality factor of the 4- $\mu m$-plate gold electrode however fluctuates a while and then continues to increase with the further increase of electrode height. The $\mathrm{Q}$ factor behaviour difference of these HARE-LWR configurations is possibly linked to their different electromechanical coupling factor $\left(K^{2}\right)$ changing trends as shown in Fig. 6c. The coupling factor is calculated by the following equation [28]:

$$
K^{2}=\frac{\pi^{2}}{4}\left(\frac{f_{s}}{f_{p}}\right) \frac{f_{p}-f_{s}}{f_{p}}
$$

where $f_{s}$ and $f_{p}$ are the resonance and anti-resonance frequencies, respectively. The increase, decrease or fluctuation of the quality factor are inversely linked to the variation of $K^{2}$. This is because the bandwidth of the resonator is inversely proportional to $K^{2}$. As a result, the quality factor improves because it is inversely proportional to the bandwidth.

To evaluate the sensing resolution of the device, we define the figure of merit (FOM) of the device as the mass sensitivity multiplied by the quality factor $\left(F O M=S_{m} \times Q\right)$. The calculated FOM is shown in Fig. 6d. It can be found that the FOMs of all the HARE-LWRs increase rapidly and reach maximums at the beginning due to the simultaneous increase of the quality factor and the mass sensitivity. Table II summarises the maximum FOM of all the HARE-LWRs and their corresponding electrode height and operating frequency. After the rapid increase, the FOMs of the 4- $\mu m$-plate HARE-LWRs based on nickel, copper and platinum decrease because the decrease of mass sensitivity. In contrast, the quality factor of the 2- $\mu m$-plate $\mathrm{Ni}$-electrode and the 4- $\mu m$-plate $\mathrm{Cu}$-electrode HARE-LWRs keep a saturated level as the decrease of mass sensitivity is compensated by the evident increase of quality factor. In reality, this FOM saturation may not happen as the quality factor increase at large electrode height can be less than the simulated results because higher electrodes can accumulate more physical defects in the fabrication process which will compromise the quality factor. It is thus better to choose an electrode height that is just high enough to fully suppress the acoustic radiation to achieve the best FOMs for all the HARELWR configurations.

\section{Comparison between HARE-LWR and SH-SAW}

To demonstrate the advantage of our design, we compared the device with conventional shear mode SAW biosensors based on SH-SAW. As the operation frequency affects the mass sensitivity, the devices in comparison are scaled to 
TABLE II

MAXIMUM FOM OF THE HARE-LWRS

\begin{tabular}{|c|c|c|c|c|}
\hline $\begin{array}{c}\text { Electrode } \\
\text { Material }\end{array}$ & $\begin{array}{c}t_{\text {plate }} \\
(\mu \mathrm{m})\end{array}$ & $\begin{array}{c}F O M_{\max } \\
\left(\mathrm{Hz} /\left(\mathrm{ng} \cdot \mathrm{cm}^{2}\right)\right)\end{array}$ & $\begin{array}{c}h_{\text {ele-max }} \\
(\mu \mathrm{m})\end{array}$ & $\begin{array}{c}f_{\max } \\
(\mathrm{MHz})\end{array}$ \\
\hline $\mathrm{Ni}$ & 2 & $3.75 \times 10^{6}$ & 2.2 & 129.96 \\
$\mathrm{Ni}$ & 4 & $4.64 \times 10^{6}$ & 4.1 & 137.67 \\
$\mathrm{Au}$ & 4 & $4.53 \times 10^{6}$ & 1.8 & 138.03 \\
$\mathrm{Cu}$ & 4 & $6.97 \times 10^{6}$ & 3.6 & 131.7 \\
$\mathrm{Pt}$ & 4 & $3.23 \times 10^{6}$ & 1.8 & 139.7 \\
\hline
\end{tabular}

proper wavelengths so that they all work around $133 \mathrm{MHz}$. The electrode thickness of the SH-SAW resonators was set to $0.02 \lambda$. The same polymer, PMMA, with the thickness of $0.02 \lambda$, is used to simulate their mass sensitivity. Table III summarizes the simulated performance data of all the devices in comparison. For HARE-LWR, the 4- $\mu m$-plate $\mathrm{Cu}-$ electrode configuration is chosen as it shows the best FOM. Fig. 6e shows the resonance frequency shifts of the SHSAW resonator based on $38^{\circ}$ Y-cut lithium tantalate when the density of the PMMA film is increased by one and two percent. Both $38^{\circ}$ Y-cut lithium tantalate (LT38) and AT-cut quartz (QAT) are used as the SH-SAW substrates. Lithium tatalate is reported to be one of the best piezoelectric substrate for SH-SAW because of its small dielectric mismatch with water, which reduces the acoustic attenuation [20], [29]. Quartz is used as SH-SAW substrate as it is reported to have higher mass sensitivity [30]. The FEM simulation results in Table III confirm that the mass sensitivity of SH-SAW based on AT-cut quartz $\left(25.9 \mathrm{~Hz} /\left(\mathrm{ng} / \mathrm{cm}^{2}\right)\right)$ is indeed higher than that of $38^{\circ}$ Y-cut lithium tantalate $\left(13.3 \mathrm{~Hz} /\left(\mathrm{ng} / \mathrm{cm}^{2}\right)\right)$. Nevertheless, their mass sensitivity is both lower than the maximum sensitivity of the 4- $\mu m$-plate $\mathrm{Cu}$-electrode HARELWR $\left(63.9 \mathrm{~Hz} /\left(\mathrm{ng} / \mathrm{cm}^{2}\right)\right)$. Although quartz substrate shows higher mass sensitivity, it is a less favorable substrate for $\mathrm{SH}-$ SAW because it has a very low relative dielectric constant $\left(\epsilon_{r}=3.8\right)$. This results in a large dielectric mismatch with water $\left(\epsilon_{r}=80\right)$ that leads to strong acoustic attenuation and thus low quality factor resonance in water [20]. The simulated quality factor of SH-SAW on AT-cut quartz (57) is a lot lower than that on lithium tantalate substrate (3881). Despite the higher mass sensitivity, the FOM of SH-SAW on quartz is significantly lower than that of lithium tantalate due to the lower quality factor on quartz. When compared with the 4$\mu m$-plate $\mathrm{Cu}$-electrode HARE-LWR, the quality factor of SHSAW based on lithium tantalate is still 28.3 times lower. This is because of the strong acoustic radiation in liquid as shown in Fig. 6f. Although the shear polarized plate motion of the SH-SAW resonator can minimize the mechanical energy transferred to the water molecules, the acoustic radiation is not eliminated as the wave velocity is above the radiation limit. The FOM of the SH-SAW based on lithium tantalate is 135 times lower than the maximum FOM of the 4- $\mu m$-plate $\mathrm{Cu}$-electrode HARE-LWR. The above comparison shows the superior performance of the proposed HARE-LWR compared to the SH-SAW resonators.
TABLE III

COMPARISON OF THE HARE-LWR AND THE SH-SAW BIOSENSORS

\begin{tabular}{|c|c|c|c|c|c|}
\hline Device & $S_{m}{ }^{\mathrm{a}}$ & $Q$ & FOM & $\lambda(\mu \mathrm{m})$ & $f(\mathrm{MHz})$ \\
\hline HARE-LWR & 63.9 & $1.10 \times 10^{5}$ & $6.97 \times 10^{6}$ & 10 & 131.7 \\
SH-SAW-LT38 & 13.3 & 3881 & $5.16 \times 10^{4}$ & 30 & 134.3 \\
SH-SAW-QAT & 25.9 & 57 & 1476 & 38 & 134 \\
\hline
\end{tabular}

\section{Discussion}

Except the above theoretical investigations, other factors can also affect the performance of the HARE-LWR biosensor. One of it is the fluid motion produced by the movement of the HAREs and the piezoelectric plate. In a lamb wave delay line configuration, it was reported that the particles can be moved towards a fixed direction if only one side of the IDT is stimulated [31]. This constant particle movement can change the regional concentration of the target analytes and affect the sensor operation. However, the particles are trapped instead of being moved when a standing wave is formed by turning on both sides of the IDTs. The proposed HARE-LWR works in a single port configuration that also generates a standing wave and thus has the same trapping effect on the particles. As the particle trapping does not change the regional concentration of the analytes, the fluid motion generated in the HARE-LWR thus does not affect its sensing performance.

Another factor that can affect the HARE-LWR's performance is the damping caused by the electrodes. It was reported that the electrode material can affect the insertion loss of conventional SAW filters [32]. One might question that using HAREs can increase the damping caused by the electrodes and thus decrease the quality factor of the HARE-LWR. This is true for the HARE-LWR operating in air as the electrode induced damping can be a significant part of energy loss. However, in the case of the HARE-LWR biosensor operating in liquid, the damping caused by the electrode is much less than the damping through acoustic radiation. In fact, the HARELWRs without sufficient electrode height cannot resonate in water because of the strong damping caused by acoustic radiation. Although the HARE increases the energy loss in the electrodes, it is a necessary trade-off to enable the operation of the device in water. We compared the quality factor impact caused by the electrode damping and the acoustic radiation through the simulation of a SH-SAW based on $36^{\circ} \mathrm{Y}$ lithium niobate whose aluminum electrode is with an isotropic loss factor $\left(\eta_{s}\right)$ of 0.01 . SH-SAW is chosen for the comparison as it can operate in liquid with acoustic radiation. The simulation results shows that the SH-SAW operate in water with the electrode damping has a quality factor of 3593 . If we set the damping of the electrode to zero $\left(\eta_{s}=0\right)$, the quality factor becomes 3901, which means the electrode damping introduced $8 \%$ of quality factor drop for the SH-SAW. If we let the same device with electrode damping work in air, the quality factor becomes 43680, which means the acoustic radiation results in 11.2 times quality drop. This comparison proves that acoustic radiation is a much stronger damping source than the loss in the electrodes. The simulated spectrum of the quality factor in this comparison are given in Fig. S5 of supporting information. 


\section{SUMMARY}

A HARE-LWR biosensor with high in-liquid quality factor and mass sensitivity is proposed. Although conventional $A_{0}$ mode Lamb wave biosensors can also achieve non-radiative operation in water by reducing their plate thickness to a very thin level, they become very fragile and thus not suitable for practical use. In contrast, the proposed HARE-LWR uses HAREs to slow down the wave while allowing for the use of thick plates, which significantly improve the structural reliability of the device. Through FEM simulations, we analyzed the slowing down behavior of the Lamb wave in HARE-LWR resonators. It was found that the resonator will enter a semileaky region after its acoustic velocity is slowed down to just below the sound velocity of water. The device is still operational in this semi-leaky region but has a relatively low quality factor. When the electrode height is further increased, the acoustic radiation becomes fully suppressed. The quality factor of the resonator is thus dramatically improved. The mass sensitivity of the resonator also reaches its maximum just after the semi-leaky region. Further increase of the electrode height reduces the mass sensitivity due to the simultaneous decrease of the resonance frequency. To evaluate the mass sensing resolution of the sensor, we defined the FOM as the mass sensitivity multiplied by the quality factor. The FOMs of the HARE-LWRs with different electrode materials and electrode heights all reach their optimal value just after the semi-leaky region. By comparing the proposed HARE-LWR biosensor with the widely used SH-SAW biosensor working at the same frequency, it was found that the HARE-LWR provides a significant 135 times FOM improvement compared to the SH-SAW. In conclusion, the HARE-LWR biosensor combines the benefits of high mass sensitivity, high quality factor and high reliability. To our knowledge, it is the first nonradiative acoustic resonator that can work reliably in water. This work has the potential to help substantially push the performance boundary of mass-sensitive acoustic biosensors.

\section{ACKNOWLEDGMENT}

This work is funded by NPRP grant No. NPRP10-0201170315 from the Qatar National Research Fund (a member of Qatar Foundation). The findings herein reflect the work, and are solely the responsibility of the authors.

\section{REFERENCES}

[1] S. Vigneshvar, C. Sudhakumari, B. Senthilkumaran, and H. Prakash, "Recent advances in biosensor technology for potential applications-an overview," Frontiers in bioengineering and biotechnology, vol. 4, p. 11, 2016.

[2] P. Mehrotra, "Biosensors and their applications-a review," Journal of oral biology and craniofacial research, vol. 6, no. 2, pp. 153-159, 2016.

[3] V. Gaudin, "Advances in biosensor development for the screening of antibiotic residues in food products of animal origin-a comprehensive review," Biosensors and Bioelectronics, vol. 90, pp. 363-377, 2017.

[4] I. Voiculescu and A. N. Nordin, "Acoustic wave based mems devices for biosensing applications," Biosensors and Bioelectronics, vol. 33, no. 1, pp. 1-9, 2012.

[5] A. Mujahid, A. Afzal, and F. L. Dickert, "An overview of high frequency acoustic sensors-qcms, saws and fbars-chemical and biochemical applications," Sensors, vol. 19, no. 20, p. 4395, 2019.
[6] I. Mannelli, M. Minunni, S. Tombelli, and M. Mascini, "Quartz crystal microbalance (qcm) affinity biosensor for genetically modified organisms (gmos) detection,' Biosensors and bioelectronics, vol. 18, no. 2-3, pp. 129-140, 2003.

[7] C. Yao, T. Zhu, J. Tang, R. Wu, Q. Chen, M. Chen, B. Zhang, J. Huang, and W. Fu, "Hybridization assay of hepatitis b virus by $\mathrm{qcm}$ peptide nucleic acid biosensor," Biosensors and Bioelectronics, vol. 23, no. 6, pp. 879-885, 2008.

[8] N. A. Masdor, Z. Altintas, and I. E. Tothill, "Sensitive detection of campylobacter jejuni using nanoparticles enhanced qcm sensor," Biosensors and Bioelectronics, vol. 78, pp. 328-336, 2016.

[9] A. Karczmarczyk, K. Haupt, and K.-H. Feller, "Development of a qcm-d biosensor for ochratoxin a detection in red wine," Talanta, vol. 166, pp. 193-197, 2017.

[10] M. Z. Atashbar, B. Bejcek, A. Vijh, and S. Singamaneni, "Qcm biosensor with ultra thin polymer film," Sensors and Actuators B: Chemical, vol. 107, no. 2, pp. 945-951, 2005.

[11] Y. Zhang, J. Luo, A. J. Flewitt, Z. Cai, and X. Zhao, "Film bulk acoustic resonators (fbars) as biosensors: A review," Biosensors and Bioelectronics, vol. 116, pp. 1-15, 2018.

[12] Y.-C. Chen, W.-C. Shih, W.-T. Chang, C.-H. Yang, K.-S. Kao, and C.C. Cheng, "Biosensor for human ige detection using shear-mode fbar devices," Nanoscale research letters, vol. 10, no. 1, p. 69, 2015.

[13] P. Guo, J. Xiong, D. Zheng, W. Zhang, L. Liu, S. Wang, and H. Gu, "A biosensor based on a film bulk acoustic resonator and biotin-avidin system for the detection of the epithelial tumor marker mucin 1," RSC Advances, vol. 5, no. 81, pp. 66355-66359, 2015.

[14] S. Ten, U. Hashim, S. Gopinath, W. Liu, K. Foo, S. Sam, S. Rahman, C. Voon, and A. Nordin, "Highly sensitive escherichia coli shear horizontal surface acoustic wave biosensor with silicon dioxide nanostructures," Biosensors and Bioelectronics, vol. 93, pp. 146-154, 2017.

[15] F. Di Pietrantonio, M. Benetti, D. Cannatà, E. Verona, M. Girasole, M. Fosca, S. Dinarelli, M. Staiano, V. Marzullo, A. Capo et al., "A shear horizontal surface acoustic wave biosensor for a rapid and specific detection of d-serine," Sensors and Actuators B: Chemical, vol. 226, pp. 1-6, 2016.

[16] J. Ji, C. Yang, F. Zhang, Z. Shang, Y. Xu, Y. Chen, M. Chen, and X. Mu, "A high sensitive sh-saw biosensor based $36^{\circ}$ yx black litao3 for labelfree detection of pseudomonas aeruginosa," Sensors and Actuators B: Chemical, vol. 281, pp. 757-764, 2019.

[17] M. Hussain, F. Rupp, H. P. Wendel, and F. K. Gehring, "Bioapplications of acoustic crystals, a review," TrAC Trends in Analytical Chemistry, vol. 102, pp. 194-209, 2018.

[18] S. Fanget, S. Hentz, P. Puget, J. Arcamone, M. Matheron, E. Colinet, P. Andreucci, L. Duraffourg, E. Myers, and M. Roukes, "Gas sensors based on gravimetric detection - a review," Sensors and Actuators B: Chemical, vol. 160, no. 1, pp. 804-821, 2011.

[19] F. Gao, F. Boussaid, W. Xuan, C.-Y. Tsui, and A. Bermak, "Dual transduction surface acoustic wave gas sensor for voc discrimination," IEEE Electron Device Letters, vol. 39, no. 12, pp. 1920-1923, 2018.

[20] K. Länge, B. E. Rapp, and M. Rapp, "Surface acoustic wave biosensors: a review," Analytical and bioanalytical chemistry, vol. 391, no. 5, pp. 1509-1519, 2008.

[21] R. White and S. Wenzel, "Fluid loading of a lamb-wave sensor," Applied physics letters, vol. 52, no. 20, pp. 1653-1655, 1988

[22] S. W. Wenzel and R. M. White, "A multisensor employing an ultrasonic lamb-wave oscillator," IEEE Transactions on Electron Devices, vol. 35 , no. 6 , pp. $735-743,1988$

[23] B. Costello, B. Martin, and R. White, "Ultrasonic plate waves for biochemical measurements," in Proceedings., IEEE Ultrasonics Symposium,. IEEE, 1989, pp. 977-981.

[24] M. Vellekoop, G. Lubking, P. Sarro, and A. Venema, "Integrated-circuitcompatible design and technology of acoustic-wave-based microsensors," Sensors and Actuators A: Physical, vol. 44, no. 3, pp. 249-263, 1994.

[25] V. Laude, L. Robert, W. Daniau, A. Khelif, and S. Ballandras, "Surface acoustic wave trapping in a periodic array of mechanical resonators," Applied physics letters, vol. 89, no. 8, p. 083515, 2006.

[26] Q.-M. Wang, X.-H. Du, B. Xu, and L. E. Cross, "Electromechanical coupling and output efficiency of piezoelectric bending actuators," IEEE transactions on ultrasonics, ferroelectrics, and frequency control, vol. 46, no. 3, pp. 638-646, 1999.

[27] V. Yantchev and I. Katardjiev, "Thin film lamb wave resonators in frequency control and sensing applications: a review," Journal of Micromechanics and Microengineering, vol. 23, no. 4, p. 043001, 2013.

[28] A. Konno, M. Sumisaka, A. Teshigahara, K. Kano, K.-y. Hashimo, H. Hirano, M. Esashi, M. Kadota, and S. Tanaka, "Scaln lamb wave 
resonator in ghz range released by xef 2 etching," in 2013 IEEE International Ultrasonics Symposium (IUS). IEEE, 2013, pp. 13781381.

[29] S. Shiokawa and J. Kondoh, "Surface acoustic wave sensors," Japanese journal of applied physics, vol. 43, no. 5S, p. 2799, 2004.

[30] K. Mitsakakis, A. Tsortos, J. Kondoh, and E. Gizeli, "Parametric study of sh-saw device response to various types of surface perturbations," Sensors and Actuators B: Chemical, vol. 138, no. 2, pp. 408-416, 2009.

[31] R. Moroney, R. White, and R. Howe, "Fluid motion produced by ultrasonic lamb waves," in IEEE Symposium on Ultrasonics. IEEE, 1990, pp. $355-358$

[32] C. Johnsen and J. Henderson, "Improved metallization for saw filters on linbo 3," in IEEE 1986 Ultrasonics Symposium. IEEE, 1986, pp. 97-102.

Feng Gao received his B.S. degree in electronic science \& technology from Zhejiang University, Hangzhou, China, and Ph.D. degree in electronic and computer engineering from The Hong Kong University of Science and Technology (HKUST), Hong Kong, in 2014 and 2018, respectively. He joined as a post-doctoral research fellow with HKUST in 2018. In 2019, he joined Hamad Bin Khalifa University, Doha, Qatar as a post-doctoral research fellow. His research interests include biosensors, gas sensors, acoustic wave devices and phononic crystals.

Aisha Al-Qahtani received her B.S. degree in electrical \& computer engineering from Texas A\&M University at Qatar and M.S. degree in biological \& biomedical science from Hamad Bin Khalifa University (HBKU), Doha, Qatar in 2014 and 2017, respectively. She is now pursuing her Ph.D. in biological \& biomedical science at HBKU. Her research interest are applying electrical engineering in the biomedical field.

Abdelkrim Khelif received his M.S. in fundamental physics and Ph.D. in Material sciences from the University of Lille, France in 1993 and 1998, respectively. He joined the FEMTO-ST Institute in 2002 as a full-time researcher at Centre National de la Recherche Scientifique (CNRS) to develop phononic crystals and acoustic metamaterials. He has extensive experience in design, optimization, and simulation of phononic structures. Due to his achievements in the field of phononic crystal, he has been involved as the symposium session chair, technical committee member, and invited speaker in several conferences in the last 5 years. He is also a frequent reviewer in this field for multiple physics and acoustic journals (Physical Review Letter, B, E, Applied Physics Letters, Journal of Applied Physics, and so on). He has more than 80 journal papers and several invited, and contributed conference and seminar presentations. He also has 4 patents in phononic crystal and is the recipient of Bronze medal awards from CNRS for his work in phononic crystal in 2007.

Farid Boussaid received his M.S. and Ph.D. degrees in microelectronics from the National Institute of Applied Science, Toulouse, France, in 1996 and 1999, respectively. He joined as a post-doctoral research fellow with Edith Cowan University, Perth, Australia, and a member of the Visual Information Processing Research Group in 2000. He joined The University of Western Australia, Crawley, Australia, in 2005, where he is currently a professor. His current research interests include gas sensors, electronic noses, smart CMOS vision sensors and image processing.
Sarah Benchabane received her M.S. degree in Optics and Optoelectronics from the University of Saint-Etienne, France in 2003. She then received her Ph.D. degree in Engineering Sciences from the Université de Franche-Comté in France in 2006. From 2007 to 2008, she was a postdoctoral research fellow the Institut de Ciencies Fotoniques (ICFO) in Barcelona (Spain). In 2008, she was appointed a CNRS research Scientist at FEMTO-ST. Her current research interests include microsonic phononic crystals, surface acoustic wave devices, micro-nano-systems and acousto-optical interactions.

Yu Cheng received her M.Phil. degree in advanced materials engineering and Ph.D. degree in civil and environmental engineering from The Hong Kong University of Science and Technology, Hong Kong. Her research interests include structure health monitoring, process and product quality control, capacitive sensors, non-destructive evaluation and finite element analysis.

Omar El-Agnaf received his Ph.D. in Biochemistry in 1997 at the Queen's University Belfast, UK. From 1997 to 1999, he worked as a post-doctoral scientist at the School of Biology and Biochemistry, Queen's University Belfast. Following this, he undertook an additional two-year post-doctoral position at St. George's Medical School in London. In 2001, he was awarded a research fellowship from the Parkinson's Disease Society-UK to establish his research group at Lancaster University. After completing his research fellowship in 2004, he was invited to join the College of Medicine at UAE University and was appointed to the Biochemistry Department. In September 2014, he joined HBKU at Qatar Foundation, and in 2016 he was appointed Acting Executive Director of the Qatar Biomedical Research Institute. He is frequently invited as a speaker at international scientific and clinical meetings and is currently a member of the editorial boards of several international journals. He has published nearly 120 refereed articles, with citations exceeding 9,000 (to date), and possesses an h-index of 52.

Amine Bermak received his M.S. and Ph.D. degrees in electrical and electronic engineering from Paul Sabatier University, Toulouse, France, in 1994 and 1998, respectively. In 2002, he joined the Electronic and Computer Engineering Department, The Hong Kong University of Science and Technology (HKUST), where he was a professor and the ECE associate head for research and postgraduate studies. Over the last decade, He has acquired a significant academic and industrial experience. He is currently with Hamad Bin Khalifa University, Qatar Foundation, Qatar. He has published over 250 articles in journals, book chapters, and conference proceedings, and designed over 30 chips. He has served on the editorial board of the IEEE Transactions on Very Large Scale Integration Systems, the IEEE Transactions on Biomedical circuits and Systems, the Journal of Low Power Electronics and Applications, and Frontiers in Neuromorphic Engineering. He is an IEEE fellow and distinguished lecturer. 